\title{
The character in the hole experiment: Interaction of forward and backward masking of alphabetic character recognition by dynamic visual noise (DVN)
}

\author{
WILLIAM R. UTTAL ${ }^{1}$ \\ THE UNIVERSITY OF MICHIGAN ${ }^{2}$
}

In the present study we consider the question of interaction between the forward and backward masking effects of dynamic visual noise (DVN) on the recognition of alphabetic characters composed of the same type of dots as those composing the DVN. The results of our experiments indicate that the two effects strongly interact so that both the duration and magnitude of the combined effect is far greater than would have been predicted by an algebraic model. Speculations about possible explanatory assumptions are presented.

In a recent paper (Uttal, 1969), the masking effects of dynamic visual noise (DVN) on the recognition of alphabetic characters were measured. DVN was defined in that experiment as a series of dots plotted at random locations in a two-dimensional space at periodic intervals. The alphabetic characters were composed of geometric patterns of the same sort of dot composing the DVN. The major advantage of this method is that there is little neurological or transductive interaction among the dots of the character and those of the DVN, either in terms of overlapping stimulation of the same retinal receptors, or in terms of possible lateral inhibitory interaction in the periphery. Due to the absence of these peripheral effects we had predicted that the masking would be less than that found with diffuse flashes of light or continuous patterns. The results of the earlier study confirmed this prediction. The maximum persistence of masking with either leading or trailing DVN turned out to be about $40 \mathrm{msec}$ as opposed to durations of $100-200 \mathrm{msec}$ in the usual masking paradigm using continuous figures. This result confirms the notion that both forward and backward visual masking are probably complex mixtures of several different effects.

An important related question now arises: How do forward and backward masking effects of DVN combine when the character is both preceded and followed by masking dots? The experimental paradigm that answers this question is one in which the character is placed in a "temporal hole" between two bursts of DVN.

There were two possible outcomes of the character in the hole experiment. The effects of leading and trailing DVN could combine in some simple algebraic fashion. Addition or multiplication of the effect of each alone in either a linear or some subtly weighted fashion might then have predicted the combined effect. Alternatively, they could interact in a more complex fashion that cannot be described simply by algebraic rules, but involving discontinuities or actual changes in the response curves. As we shall see below, an examination of the data made it quite clear that there are strong and interesting interactions produced when both leading and trailing DVN are used simultaneously. These results are consistent with the analogous results in audition reported by Pollack (1964) or Bilger (1959) that also showed deviation from additive models. In the present case the discrepancy is even greater because substantial masking occurs at intervals at which any kind of algebraic model would have predicted there should have been none. Thus, rather than emerging with a set of algebraic rules describing some ideal features of the stimuli, we now demonstrate another situation in which the information-processing capacity of the $O$ defines the effects described. The purpose of this present study is to determine the nature of the interaction between leading and trailing noise and to propose some possible explanations of that interaction.

\section{Subjects}

\section{METHOD}

Six to eight male and female Ss with normal vision contribute data to each point plotted in our data figures. In all cases the data we report have been pooled, so information concerning individual differences is lost. All Ss had 2 or 3 days of pretraining in an experimental configuration in which the characters were easily recognizable, and they were performing at the $95 \%$ (or above) level of correct character recognition for that situation when the data reported here were collected.

\section{Apparatus}

The entire experiment was under the control of a digital

\section{INTERDOT INTERVAL}

$15 \mathrm{msec}$

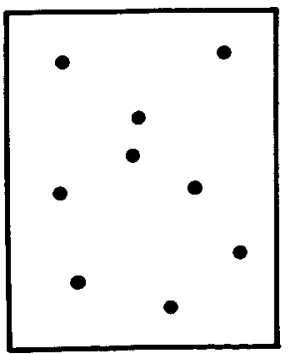

$5 \mathrm{msec}$

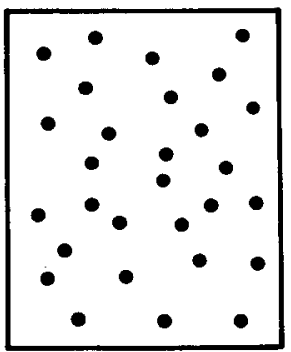

I msec

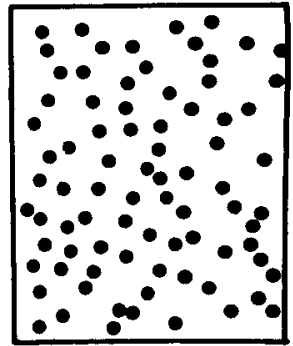

Fig. 1. Artist's impressions of three different densities of dynamic visual noise (DVN). The interdot interval is the specific metric used in our experiments to define the DVN level. Each dot is present for less than a millisecond, but the integrating properties of the eye give the appearance of a field of many dots, the actual number dependent upon the interdot interval and the eye's integrating period. 

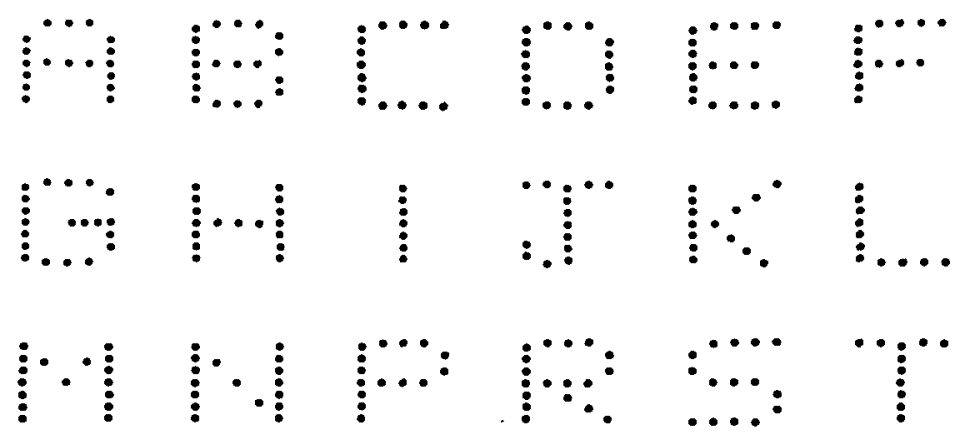

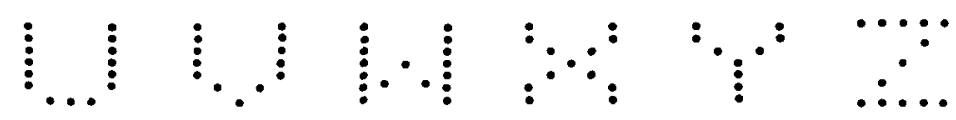

Fig. 2. The 24-character alphabet used in the present experiments.

computer that was fully dedicated to this single application because of high data rates and precise time measurements involved in the stimulus generation process. A complete description of the character font, the characteristics of the DVN, the $\mathrm{S}$ cubicle, and the display geometry is presented in the previous paper (Uttal, 1969). In the present paper we shall recapitulate only the most important details. Figure 1 shows the appearance (artist's impressions) of the DVN at three interdot intervals, while Fig. 2 shows the character set used in the experiment.

All stimulus information was displayed on the face of a CRT with an ultrashort persistence P-15 phosphor. The persistence of the light emitted from the screen is such that it is reduced to $.1 \%$ of its initial value in less than 50 microsec. Because the eye integrates over time, a considerable number of dots is seen on the screen at any instant even though the physical persistence of a given dot on the CRT is short. As a preliminary rule of thumb we have assumed that the duration of the visual integrating period is about $100 \mathrm{msec}$, and the number of dots perceptually present at any instant is therefore approximately equal to $100 \mathrm{msec}$ divided by the interdot interval.

The S's face was guided by a forehead constraint to a position $14 \mathrm{in}$. from the face of the CRT. Noise was displayed in an area that measured $9.2 \mathrm{deg}$ by $6.1 \mathrm{deg}$ of visual angle, and the characters were $4.0 \mathrm{deg}$ by $3.0 \mathrm{deg}$ of visual angle in size. The characters were jittered in position on the vertical axis to avoid pseudorecognition by parts. The stimulus alphabet used did not include $O$ and $Q$, the two being almost indistinguishable with the character font we were using.

\section{General Procedure}

During each session the order of character presentation was randomized by random number generators in the computer program. However, within each daily session the experimental configuration and the level of the DVN were kept constant. Two Ss ran as a team in each experimental session, each serving half the time as the experimental $S$ and half the time as a transcriber typing the intercommunicated responses of his partner into a keyboard for direct entry into the magnetic tape data storage files of our computer. The transcriber $S$ also fed back knowledge of results to the experimental $S$ by telling him the correct character when, and only when, the experimental $S$ had made a mistake.

Data were accumulated in separate files of the computer's magnetic tape memory for each $S$ for each session. At the end of the day, the data of all Ss were completely analyzed and pooled by the application of two analysis programs. DAP 1 , the first analysis program, summarized the results for each $S$, tabulating errors, and giving the total number of presentations, the number correct, and percentage correct for each of the 24 characters used in our experimental alphabet. DAP 2, the second analysis program, pooled all data from all $S s$ and gave the same information as DAP 1 for the total data of each day. The percentage correct score given by DAP 2 was, therefore, without further processing, the value of a specific point plotted on each of our graphs. Usually the results of 2,000 trials were pooled for each point. However, in some of the more difficult conditions, the Ss slowed their rates considerably, and in some cases as few as 1,000 trials constituted the sample size for a single data point.

In both of the experiments to be reported in this present paper, the initial warning signal to the $S$ indicating that the computer was ready was the beginning of the DVN itself. The S was instructed to release the programmed sequence of the character in the hole (DVN-delay-character-delay-DVN) by pushing a hand-held button when he was ready and to proceed at a "rapid" pace. The computer detected the button press and then added $1 \mathrm{sec}$ of leading DVN before timing out the first delay period between the leading noise and the character. This additional second guaranteed that the $S$ did not reduce the leading masking period to an ineffectually short duration by anticipating the onset of the DVN. Following the first delay all the points of the character were then displayed in less than $.4 \mathrm{msec}$. The second delay period was then timed out by the computer, and then the trailing DVN began and was maintained for $1 \mathrm{sec}$. The sum of the two delay periods, therefore, defined the duration of the temporal hole in which the character was positioned.

In the first experiment reported in this paper, the duration of the temporal hole-the independent variable-was varied, and the percentage of the total number of characters presented that were correctly identified was measured as the indicator of the masking effects of the combined leading and trailing DVN. Hole sizes of $200,150,100,80,60,40,20$, and $10 \mathrm{msec}$ were used. In all cases in this experiment the character was kept positioned at the temporal center of the hole between the two bursts of DVN. This experiment was repeated with two values of DVN, $3 \mathrm{msec}$ and $1 \mathrm{msec}$, selected because of their special significance in our earlier report (Uttal, 1969). This metric of DVN-value in milliseconds-is the measure of the time between the plotting of sequential random points. As we noted above, such a value is, of course, very much confounded by the persistency of the visual image in the S's short-term visual store.

In the second experiment, the hole was kept constant at a duration of $60 \mathrm{msec}$, a period slightly less than twice the measured period in which the leading or trailing DVN alone was effective as a mask. However, in this case the position of the character in the hole was varied in 10-msec steps. In our figures, 


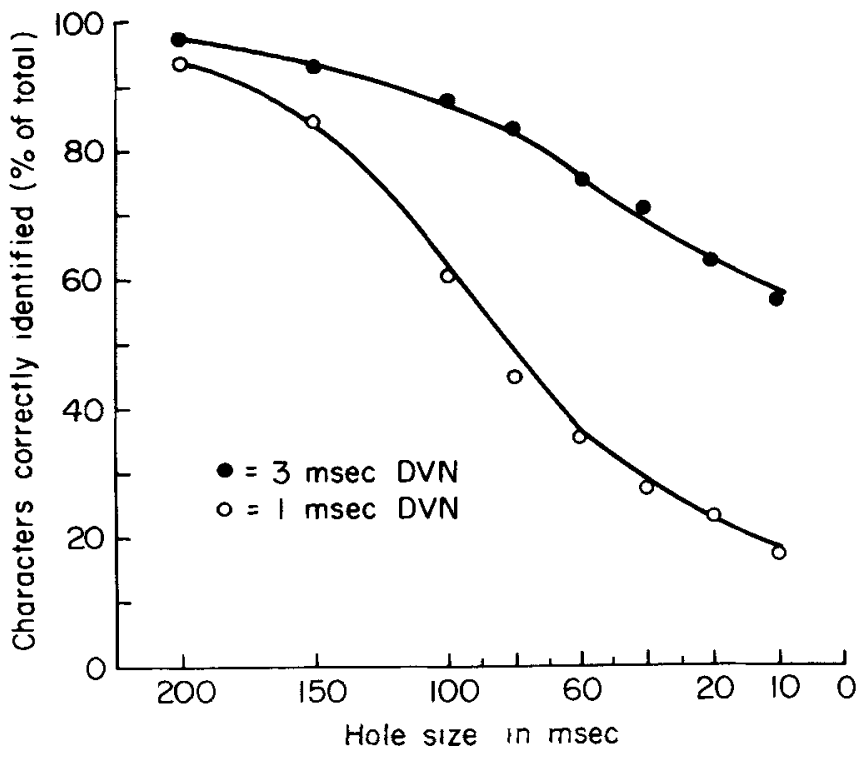

Fig. 3. The results of the first experiment in which the hole size was varied and the character was always presented at the center of the temporal hole. This curve shows that the interaction of the forward and backward masking leads to masking at intervals greater than would have been predicted on the basis of Fig. 6.

the sum of each pair of numbers (e.g., 50-10) designates the duration of the hole (in milliseconds), the first number indicating the duration of the first delay and the second number the duration of the second delay. Values of $0-60,10-50,20-40$, $30-30,40-20,50-10$, and 60-0 were used. Our hypothesis was that if the interaction between the two bursts of DVN had been representable by an algebraic expression, then at the midpoint almost no masking should have been observed, and the curve should have been bowed up in the middle. Even if the combination of the two masking bursts is weighted in some manner, then minimum masking should have occurred when the character was centered. As we shall see below, this is exactly what does not occur, and the interaction must be assumed to be more complex.

In the second experiment the values of DVN used were 3 and $2 \mathrm{msec}$, rather than 3 and $1 \mathrm{msec}$. This was necessitated by the way in which our experiment was designed. At $1 \mathrm{msec}$, Ss were performing continuously at very low levels (about 35\%), and we did not wish to have our well-trained $S$ s exposed to this low level of success for the 7 days that this experiment required. As an altemative we ran the $1-\mathrm{msec}$ case with a hole size of $100 \mathrm{msec}$. In this corollary experiment the character was placed at 10-90, $30-70,50-50,70-30$, and $90-10 \mathrm{msec}$.

\section{RESULTS}

Figure 3 displays the results of the first experiment in which the hole size was varied and the character was kept centered in the hole. The first major effect to be noted is that there is an effect of the DVN on character recognizability when the hole is as large as $150 \mathrm{msec}$. This is to be contrasted with the effects shown in Fig. 6, the data from our previous experiment, in which leading and trailing noise were presented separately. In that case the effects of DVN were undetectable at intervals as small as $35-40 \mathrm{msec}$ before or after the DVN. Thus, the two cases produce quite different initial estimates for the persistence of the masking effects. Similarly, the absolute levels in the present experiment are all lower than would have been predicted by an independent combination of the two effects. The performance of the $S$ declines steadily as the hole size decreases, asymptoting towards the values measured in the previous experiment when there was no hole and the character was centered in continuous DVN. These internal anchor points were achieved even though a new set of Ss was used for this series of experiments.

The data, parametric in terms of the DVN levels, produce no special surprises. One-millisecond DVN produces a considerably greater masking effect than the less dense 3-msec DVN, and decreasing the size of the hole decreases the performance level of the Ss.

Figures 4 and 5 display the results of the second experiment in which the character was moved about in a fixed-length hole. Figure 4 shows the results for a hole size of $60 \mathrm{msec}$ with DVN levels of 3 and 2 msec, respectively. The initial surprising result in this case was that the position of the character in the hole does not affect recognizability of the character to any substantial degree. The only exception to this generality is that there does appear to be a slight dip when the character is close to the trailing noise. Though it is difficult at this stage (because we cannot yet describe the more complex general interaction between forward and backward masking) we can understand how the dip could occur in a qualitative way. According to Fig. 6 , the effects of the leading noise last slightly longer than those of the trailing noise. Thus they would tend to add to the masking at delays between the DVN and the character longer than those at which the trailing noise was effective. Thus, characters close to the trailing noise would be masked by the trailing noise as well as by some small vestige of the leading noise, while a character close to the leading noise would be masked by the leading noise but would not fall within the effective range of the trailing noise.

The dashed lines on Fig. 4 indicate the recognition score for a centered character in a $60-\mathrm{msec}$ hole between bursts of $3-\mathrm{msec}$ DVN from Experiment 1. The dotted and dashed line indicates the recognition score for a $60-\mathrm{msec}$ hole in $1-\mathrm{msec}$ DVN in that same experiment. The fact that there is a larger difference between 1 and $2 \mathrm{msec}$ than between 2 and $3 \mathrm{msec}$ is consistent with the data from our earlier study. This nonlinearity can be

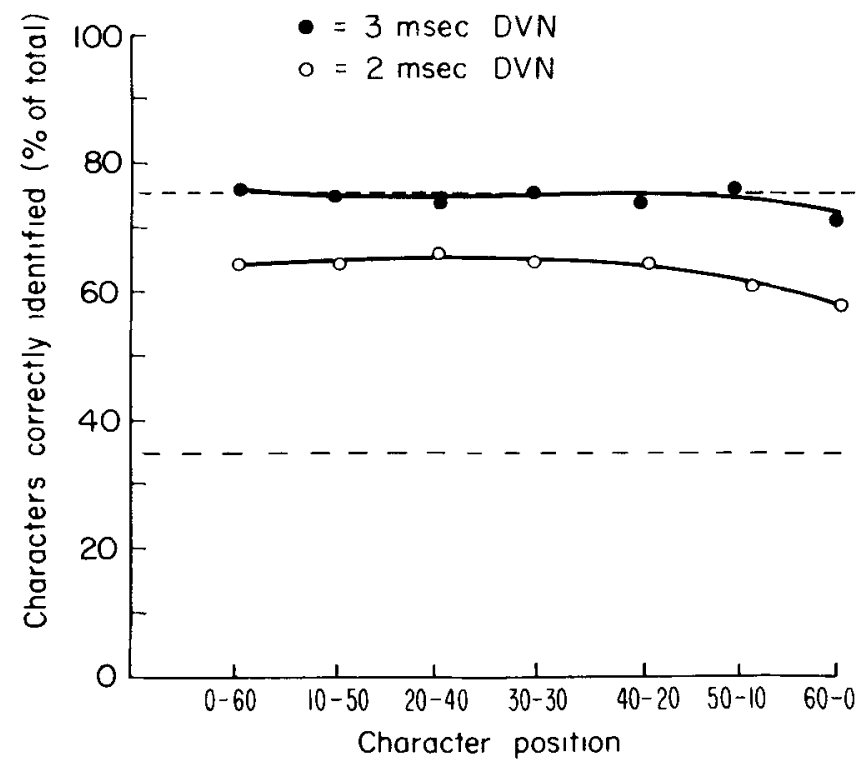

Fig. 4. The results of the second experiment showing the relatively small effect of character position in the $60-\mathrm{msec}$ hole on the recognizability of alphabetic characters. 


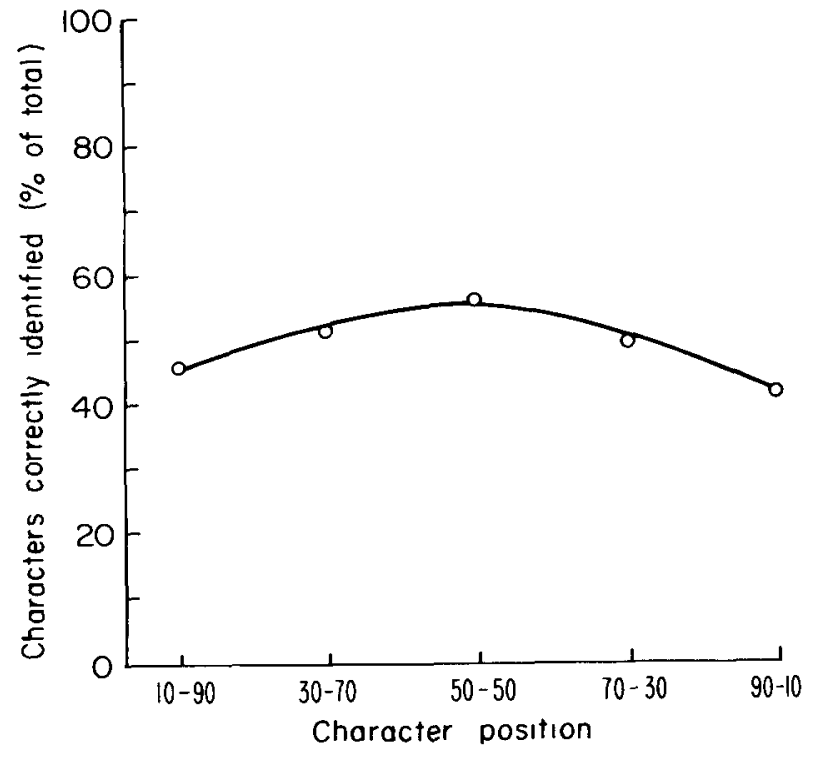

Fig. 5. The results of the corollary to the second experiment showing the slight bowing up in recognition scores when the hole is opened to $100 \mathrm{msec}$.

accounted for if one assumes an integrating period in which the number of dots is more important than the interval between the individual dots. Conversion of the millisecond measure to $n$, where $\mathrm{n}$ is the number of dots in a given integrating period, linearizes the data.

Figure 5 shows the results for that special case of Experiment 2 in which the hole size was $100 \mathrm{msec}$ and the DVN level was $1.0 \mathrm{msec}$. In this case there is a slight upward bow when the character is centrally located in the hole, indicating that the effects of the combination of leading and trailing noise is diminished when the character is $50 \mathrm{msec}$ from each. Even in this case, however, there is still considerable masking; the performance level is about $50 \%$ compared to the $95 \%$ level that would have been predicted by a model assuming strict linear combinations of forward and backward masking.

\section{DISCUSSION}

The results of the experiments reported here must be considered in a very limited context. These results do generalize and do have implications to related notions of the "psychological moment" or to "visual simultaneity." I choose, in this present paper, to emphasize the details of the persistence of the interference for sharacter recognition. Within this limited context, several important results can be identified.

First, let us consider the results of the first experiment and their inconsistency with the data of our previous experiment in which forward and backward masking were presented separately. As indicated in Fig. 6, the duration of the effect is quite brief compared to the present results. The effects of forward masking have risen to control levels for no masking at about $40 \mathrm{msec}$. Similarly recognition scores are above $90 \%$ when the trailing noise follows more than $30 \mathrm{msec}$ after the character.

In the present experiment, however, detectable masking occurs when the hole is as large as $150 \mathrm{msec}$, indicating that the effects of forward and backward masking have been extended to delays of $75 \mathrm{msec}$. The second major result of this study is that the recognition scores are not affected by character position when the hole is $60 \mathrm{msec}$ in duration. Both of these experiments support the notion that no algebraic model can account for the data. By an algebraic model we are generally referring to a mathematical description of the data that attempts to predict the data of the present experiment from that of Fig. 6. But in addition, an algebraic model also denotes a continuous function involving additivity or multiplicativity assumptions. Our results, to the contrary, state that there are psychological processes at work in this experiment that either introduce some sort of discontinuity or in some other way invalidate the measures of the function shown in Fig. 6 when leading and trailing DVN are presented together.

First let us consider in greater detail the data that suggest that there is no algebraic model capable of explaining these results. It should also be pointed out that the results of our previous experiment implicitly suggested that the effects of forward and backward masking were not independent of each other. Complete independence would have dictated that the results of the experiment in which the character was centered in a continuous burst of DVN should have been predicted from the results of independent forward and backward masking effects in accord with the well-known statistical statement for the probability of the joint occurrence of independent probabilities:

$$
P(F B)=P(F) \cdot P(B)
$$

where $P(F B)$ is the probability that a character will be seen with both forward and backward masking, $P(F)$ is the probability that

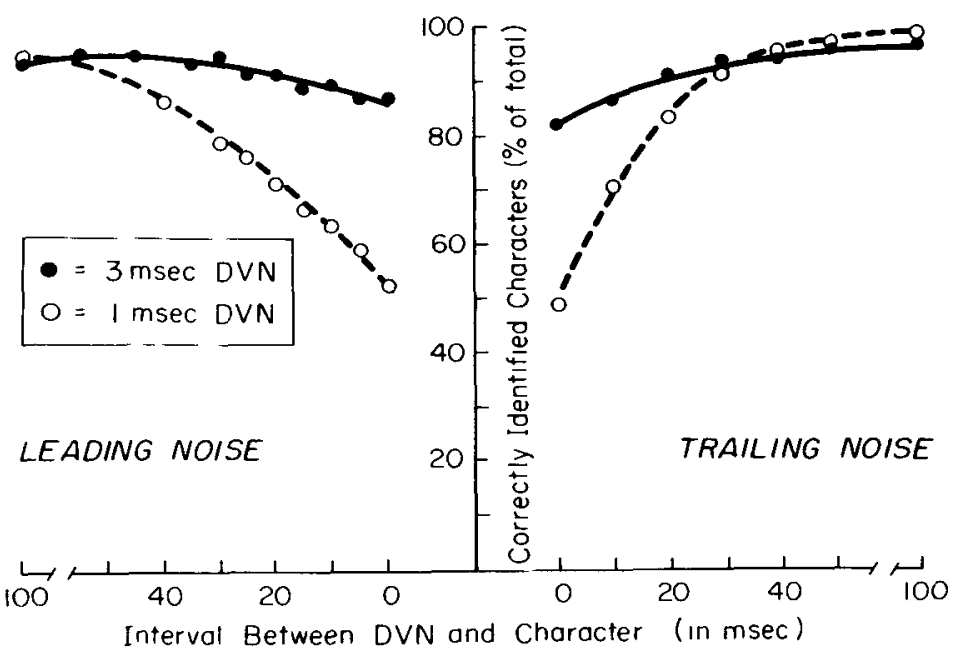

Fig. 6. The data from the previous experiment (Uttal, 1969) showing the separate masking effects of leading and trailing DVN for 1- and 3-msec interdot intervals. 
the character will be seen with forward masking alone, and $P(B)$ is the probability that the character will be seen with backward masking alone. For the $1-\mathrm{msec}$ DVN level this equation would have predicted that $\mathrm{P}(\mathrm{FB})$ (pooled data) should have been equal to .25 $[\mathrm{P}(\mathrm{F})=.5, \mathrm{P}(\mathrm{B})=.5]$ if the two masking effects were independent of each other. However, the actual score was $12.5 \%$ ( $4.4 \%$ of which could be attributed to chance alone). Thus the amplitude of the combined forward and backward masking is greater than anticipated and adds to the argument that the effects of the experiment combined nonalgebraically.

Now let us consider the second major result of this paper. The position of the character in the $60-\mathrm{msec}$ hole was shown to have no significant effect on the recognition scores. This result is also contrary to the prediction that would have been made on the basis of Fig. 6 on the basis of an assumption of independence. At the center of the 60-msec hole, little masking from either the trailing or leading DVN should have occurred. Yet the data of the corollary experiment clearly show that even at $100 \mathrm{msec}$ the expected bowing upwards at the center position is only $10 \%$. Even then this bowing only brings the recognition score up to $55 \%$-far lower than it should have been given no interaction between the forward and backward masking.

How then can the data be explained? It is the opinion of the author that the data at the present time do not permit us to specify a unique model. There are many possibilities for explanatory assumptions and perhaps (literally) an infinite number of mathematical models that could describe the results adequately. There are, however, two explanatory assumptions that, if not more intrinsically powerful than the other possibilities, are at least more obvious.

The first possibility is that the durations of the individual persistent masking effects are in fact being elongated in some manner in the combined case. That is, the persistence functions that we have measured and plotted in Fig. 6 are accurate but only for the case in which the forward or backward masking bursts of DVN are presented alone. However, they may not represent adequately the persistencies when the two masking bursts of DVN are present and interacting. Some unknown psychobiological process may actually have extended the time constants of the effects. Thus new forward and backward masking functions are introduced that then might allow algebraic assumptions to account for the differences in the data.

However, such an unknown psychobiological process is an unnecessary complication and we can consider an alternative explanatory assumption based upon the notion of a discontinuity in the S's sensitivity to the masking effects of small numbers of dots. Although our curves do not show such a discontinuity, it is clear that the sensitivity to the masking dots of the DVN could be such that below a certain number of dots, our Ss were able to read through the DVN with essentially perfect accuracy. Thus, the alternative notion that we propose is one involving the concept of a threshold density of the DVN dots. For densities below that threshold the signal-to-noise ratio is such that the S's performance is not interfered with to any degree. Densities above that threshold would be supposed to have significant measureable effects, however. This notion also implies that our experimental paradigm is not able to detect the tails of the persistence function. These tails include, of course, the short-term storage of the final dots in a forward-masking situation and the first few dots in a backward-masking situation.

According to the threshold notion the "interaction" that has been repeatedly demonstrated in the present series of experiments would then be introduced as the summation of subthreshold dot densities to give suprathreshold dot densities at intervals that previously had shown no masking effects. The second experiment in the present study (in which the hole was $60 \mathrm{msec}$ in duration) further indicates that these new effects are relatively symmetrical and almost perfectly complementary. There is only a slight dip when the character is very close to the trailing noise, due as we have indicated above, to the somewhat greater persistence of the leading noise.

This model too, however, also has its difficulties. In another experiment yet to be published ${ }^{3}$ the separate forward and backward masking paradigms were replicated with ultrahighdensity DVN. Interdot intervals of 25 microsec were used rather than the 1-, 2-, or $3-\mathrm{msec}$ intervals of this experiment. If the threshold effect is significant it would have been expected that in this yet to be fully reported case the persistence of the masking effect should have been considerably longer since sufficient numbers of dots would have been present in the tails of the visual storage periods. In fact, however, the persistence of the masking effects was only slightly, if at all, elongated. It is hard to make this result compatible with the notion of the threshold for lower dot densities. What it does say is that the pattern of persistence of the visual image is more important than the statistical estimates of the number of dots present in any psychological moment. In other words, the temporal distribution of the dots is critical and the notion of the integrating period of visual perception cannot be pushed too far as an explanation of all of the effects we have so far noted.

Finally, we must reiterate that at this time no formal explanatory model capable of describing the major results of these experiments has emerged. Further experiments now under way may give us some additional insights into the assumptions that will have to underlie such a theory. Nevertheless, the general experimental paradigm character recognition in DVN-does allow us to formulate questions in a more powerful way than previously has been available.

\section{REFERENCES}

BILGE R, R. C. Additivity of different types of masking. Journal of the Acoustical Society of America, 1959, 31, 1107-1109.

POLLACK, I. Interaction of forward and backward masking. Journal of Auditory Research, 1964, 4, 63-67.

UTTAL, W. R. Masking of alphabetic character recognition by dynamic visual noise (DVN). Perception \& Psychophysics, 1969, 6, 121-128.

\section{NOTES}

1. Address: Department of Psychology, The L niversity of Michigan, Ann Arbor, Michigan 48104.

2. The research in this paper was supported by NSF Grant GB 6093.

3. Uttal, W. R. The masking effects of ultrahigh density dynamic visual noise (DVN) on alphabetic character recognition (in preparation).

(Accepted for publication March 5, 1969.) 